\title{
Estrutura populacional e época de reprodução de Astyanax fasciatus (Cuvier) (Characidae, Tetragonopterinae) do Rio Ceará Mirim, Poço Branco, Rio Grande do Norte, Brasil
}

\author{
Hélio de Castro Bezerra Gurgel \\ Departamento de Fisiologia, Universidade Federal do Rio Grande do Norte. Caixa Postal 1511, 59072-970 Natal, Rio Grande \\ do Norte, Brasil. E-mail: helio@ufrnet.br
}

\begin{abstract}
Populational structure and breeding season of Astyanax fasciatus Cuvier (Characidae, Tetragonopterinae) from Ceará Mirim River, Poço Branco, Rio Grande do Norte, Brazil. The aim of this study was to evaluate 209 samples (100 females and 109 males) of Astyanax fasciatus (Cuvier, 1819) collected from the Ceará Mirim River, Rio Grande do Norte State, ( $5^{\circ} 37^{\prime} \mathrm{S}$ e $\left.35^{\circ} 39^{\prime} \mathrm{W}\right)$, between February 1996 and April 1997. The sex ratio was 1:1 and the the total length varied from 3.0 to $9.0 \mathrm{~cm}$, with most classes between $4.0-5.0 \mathrm{~cm}$ and 7.0-8.0 $\mathrm{cm}$. The estimated value for the parameter $(\theta=2,59)$ suggests that this specie has an alometric growing pattern. The IGS value started to increase in November-December-January, following the rainfall while the condition factor values showed.
\end{abstract}

KEY WORDS. Fish, Neotropical, reproduction.

RESUMO. Neste estudo foram capturados 209 exemplares de Astyanax fasciatus (Cuvier, 1819), sendo 100 fêmeas e

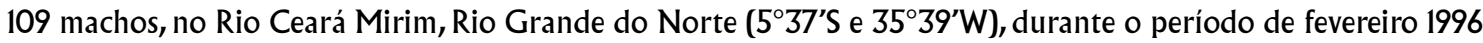
a abril de 1997. A proporção entre os sexos, não difere significativamente de 1:1, com uma leve predominância de machos. A distribuição de freqüência percentual de machos e fêmeas por classes de comprimento total, evidencia que a amplitude de variação de comprimento está compreendida entre 3 a $9 \mathrm{~cm}$, com predominância nas classes $4-5$ e 7-8 $\mathrm{cm}$. $O$ valor estimado do coeficiente angular $(\theta=2,59)$, sugere que a espécie apresenta um crescimento do tipo alométrico negativo. O IGS tende a aumentar a partir do trimestre novembro-dezembro-janeiro, alcançando seus maiores valores no trimestre seguinte, coincidindo com a elevação das médias dos valores de precipitação pluviométrica, enquanto que, o fator de condição tende a apresentar os valores médios inferiores neste período. PALAVRAS CHAVE. Peixe, neotropical.

As águas continentais contêm além de peixes nobres, uma série de outras espécies que apresentam certa importância pela ampla distribuição, volume de captura, condição de forrageio ou pesca esportiva. São peixes estritamente de água doce, com grande capacidade de adaptação, inclusive em cavernas (SANTos et. al. 1995).

Entre os peixes Characidae, o maior número de gêneros concentra-se em Tetragonopterinae, sendo Astyanax Baird \& Girrard, 1854 um dos mais ricos em espécies e o de distribuição geográfica mais ampla e diversas de suas populações apresentam, aparentemente, pouca diferenciação morfológica, ecológica e comportamental, sugerindo um grupo em especiação. Godoy (1959) os considera como elementos importantes para a manutenção do equilíbrio ecológico e salienta que apresentam o mesmo ciclo reprodutivo dos peixes de "piracema", realizando curtas migrações ascendentes na época de cheias, o que lhes proporciona o estímulo necessário para a reprodução.
GERY (1977) a descreveu como espécies de pequeno porte, onde a maioria atinge médias de $100 \mathrm{~mm}$, podendo algumas vezes alcançar $200 \mathrm{~mm}$.

Quanto à distribuição geográfica, STERBA (1973, in BARBIERI et al. 1996) diz ocorrer da América Central até a Argentina. Este grupo é popularmente conhecido, como piabas no nordeste brasileiro, lambaris no Brasil meridional e matupiris na Amazônia. Possuem importância como forrageiros de algumas espécies carnívoras (CÂMARA et al. 1991), sendo ainda utilizado como alimento por pescadores artesanais da região. Apresentam corpo alongado, nadadeiras avermelhadas, escamas com reflexos dourados, ocelo umeral difuso e faixa lateral bem nítida (VANZOLINI et al. 1964).

Os principais estudos disponíveis sobre a espécie, são os de Vanzolini et al. (1964), Rebouças (1964), BRITSKi (1964), Nomura (1975), BARBIERI et al. (1982), BARBIERI \& BARBIERI (1988) e BARBIERI et al. (1996).

Revista Brasileira de Zoologia 21 (1): 131-135, março 2004 
Astyanax fasciatus (Cuvier, 1819) figura entre as espécies mais abundantes do rio Ceará Mirim, e apesar de porte reduzido é de grande importância como elo na cadeia alimentar desse ecossistema. Assim, o presente estudo, tem como objetivo contribuir para o conhecimento de alguns aspectos da biologia dessa espécie, através da análise da estrutura em sexo e em comprimento, da relação peso total e o comprimento total, e do índice gônado-somático. Estudos dessa natureza têm sido sugeridos porque, além de esclarecer aspectos referentes à biologia de uma determinada espécie, fornecem informações valiosas sobre possíveis alterações ambientais.

\section{MATERIAL E MÉTODOS}

O material utilizado nesta pesquisa foi coletado no rio Ceará Mirim, Rio Grande do Norte $\left(5^{\circ} 37^{\prime} \mathrm{S}\right.$ e $\left.35^{\circ} 39^{\prime} \mathrm{W}\right)$ por meio de capturas mensais, durante o período de fevereiro 1996 a abril de 1997. Foram capturados 209 exemplares sendo 100 fêmeas e 109 machos, utilizando tarrafa e peneiras. Para cada exemplar registraram-se os dados de: comprimento total $\left(\mathrm{L}_{\mathrm{t}}\right)$ em $\mathrm{cm}$, peso total $\left(\mathrm{W}_{\mathrm{t}}\right)$, e o peso das gônadas $\left(\mathrm{W}_{\mathrm{g}}\right)$ em $\mathrm{g}$, seguindo-se a metodologia estabelecida por SANTos (1978).

A estrutura no que se refere à proporção entre os sexos foi estabelecida através das freqüências trimestrais de fêmeas e de machos. O teste do qui-quadrado $\left(\chi^{2}\right)$ foi aplicado com o propósito de testar as possíveis diferença entre as proporções estabelecidas, ao nível de 5\% de significância.

A estrutura em comprimento baseou-se na distribuição das freqüências percentuais trimestrais das classes de comprimento total de $1 \mathrm{~cm}$, considerando-se os sexos separados.

A relação entre o peso total $\left(\mathrm{W}_{\mathrm{t}}\right)$ e o comprimento total $\left(\mathrm{L}_{\mathrm{t}}\right)$ foi estimada para os sexos agrupados através da distribuição dos pontos empíricos destas duas variáveis e, pela dispersão destes foi possível à utilização da expressão matemática:

$W_{t}=f$. $L_{t}{ }^{q}$, onde: $\left(W_{t}\right)$ peso total em gramas; $(\phi)$ Coeficiente linear $=$ fator de condição relacionado com o grau de engorda do indivíduo; $(\theta)$ Coeficiente angular = relacionado com o tipo de crescimento do indivíduo.

Feita a transformação logarítmica das variáveis, verificou-se a existência da relação linear entre elas, transformadas através da expressão: $\ln \mathrm{W}_{\mathrm{t}}=\ln \phi+\theta \ln \mathrm{L}_{\mathrm{t}}$.

Os coeficientes $\ln \mathrm{W}_{\mathrm{t}}$ e $\ln \mathrm{L}_{\mathrm{t}}$ foram estimados pelo método dos mínimos quadrados. Calculou-se o valor do coeficiente linear de Pearson (r) para demonstrar a aderência dos pontos empíricos à reta calculada.

A existência ou não de diferenças entre a relação peso total/comprimento total, para cada sexo, foi analisada pela superposição dos gráficos de dispersão.

Para avaliar o estádio de desenvolvimento das gônadas calculou-se os valores médios trimestrais do índice gônadossomático (IGS) de fêmeas e machos que efetivamente participaram da reprodução, tendo como expressão: IGS $=\mathrm{W}_{\mathrm{g}} / \mathrm{W}_{\mathrm{t}} \cdot 100$.

Considerando que o parâmetro $\theta$, é relacionado com o tipo de crescimento do animal deve manter-se constante para a espécie, estimou-se o valor de $\theta$ para todos os exemplares, considerando-se os sexos separados. Desta forma, calculou-se o fator de condição corrigido $\left(\phi^{*}\right)$ para machos e fêmeas separadamente (BARBIERI \& SANTOS 1980) sendo expresso pela relação: $\phi^{*}=\mathrm{W}_{\mathrm{t}} / \mathrm{L}_{\mathrm{t}}{ }^{\theta}$.

Aos valores do fator de condição $\left(\phi^{*}\right)$ e do índice gônadossomático (IGS), foram associados às médias dos valores trimestrais de precipitação pluviométrica.

\section{RESULTADOS E DISCUSSÃO}

VAZzOLER (1996), considera que a proporção entre fêmeas e machos é uma informação importante para a caracterização da estrutura de uma espécie ou população, além de constituir subsídio para estudo de outros aspectos como a avaliação do potencial reprodutivo e em estimativas do tamanho do estoque. Segundo a autora, este parâmetro em peixe varia ao longo do ciclo de vida em função de eventos sucessivos, que atuam de modo distinto sobre os indivíduos de cada sexo, constituindo tática reprodutiva.

A proporção entre os sexos (Fig. 1), não difere significativamente de 1:1, numa análise envolvendo o total de exemplares, com uma leve predominância de machos. $\mathrm{Na}$ análise trimestral, as fêmeas mostram predomínio significativo nos trimestres fevereiro-março-abril de $1996\left(\mathrm{n}=100 ; \chi^{2}=10,57\right)$ e de 1997 ( $\left.n=69 ; \chi^{2}=14,44\right)$, enquanto que os machos predominam significativamente no trimestre maio-junho-julho de $1996\left(\mathrm{n}=69 ; \chi^{2}=23,00\right)$. Essa diferença no comportamento pode ser explicada pelo fato das fêmeas, devido ao peso da gônada, tornarem-se mais susceptíveis à captura.

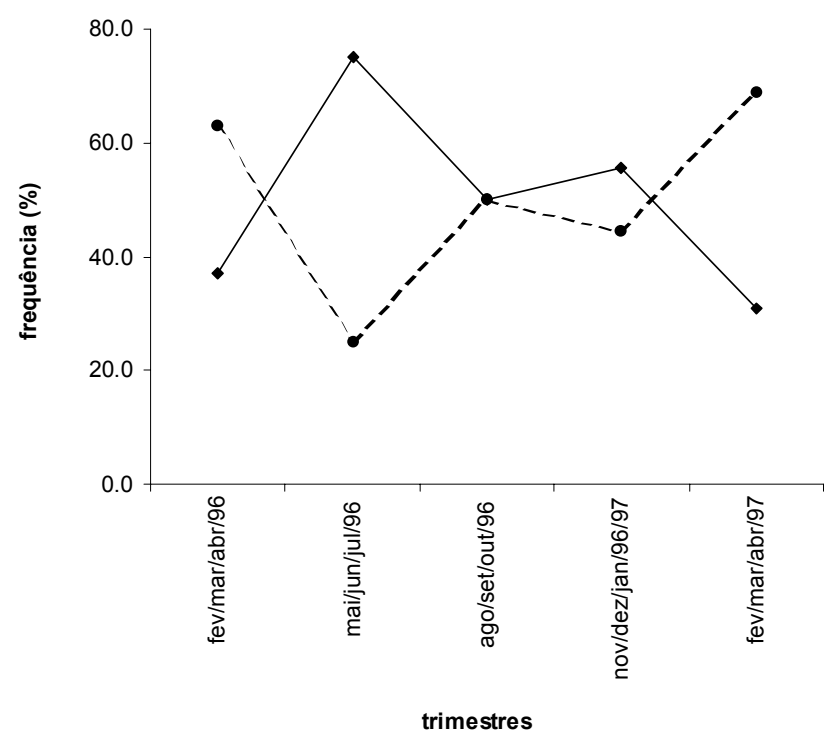

Figura 1. Distribuição trimestral das freqüências percentuais de fêmeas e machos durante o período total de estudo. 
Resultados semelhantes foram obtidos para outras espécies por Diвв et al. (1991), estudando Crenicichla lepidota Heckel, 1840, Gurgel (1995), para Metynnis cf. roosevelti Eigenmann, 1915, da lagoa Redonda, Rio Grande do Norte e Raposo \& GurgeL (2001) com Serrasalmus spilopleura Günther, 1864, da lagoa de Extremoz, Rio Grande do Norte.

Entre os fatores que poderiam influir na razão sexual, o suprimento alimentar da população foi considerado por NIKOLSKII (1969) como fator importante. Assim, segundo este autor, nas represas e rios oligotróficos há predominância de machos. As fêmeas predominam quando o alimento disponível é abundante.

A composição de uma população em classes de comprimento é uma característica que responde ao ambiente e pode variar de ano para ano, dependendo também da sua fecundidade (NiKOLSKII 1969) e pode fornecer subsídios para o estudo da determinação do equilíbrio dessa população, envolvendo estimativas das taxas de mortalidade, reprodução, recrutamento e crescimento.

As distribuições de freqüências percentuais de machos e fêmeas de $A$. fasciatus por classes de comprimento total (Fig. 2), evidenciam que a amplitude de variação de comprimento está compreendida entre 3 a $9 \mathrm{~cm}$. Observa-se a maior freqüência de fêmeas nas classes de 4-5, 5-6, 8-9 e 9-10 cm. A maior ocorrência de machos foi registrada nas classes de 3-4, 6-7 e 7-8 cm.

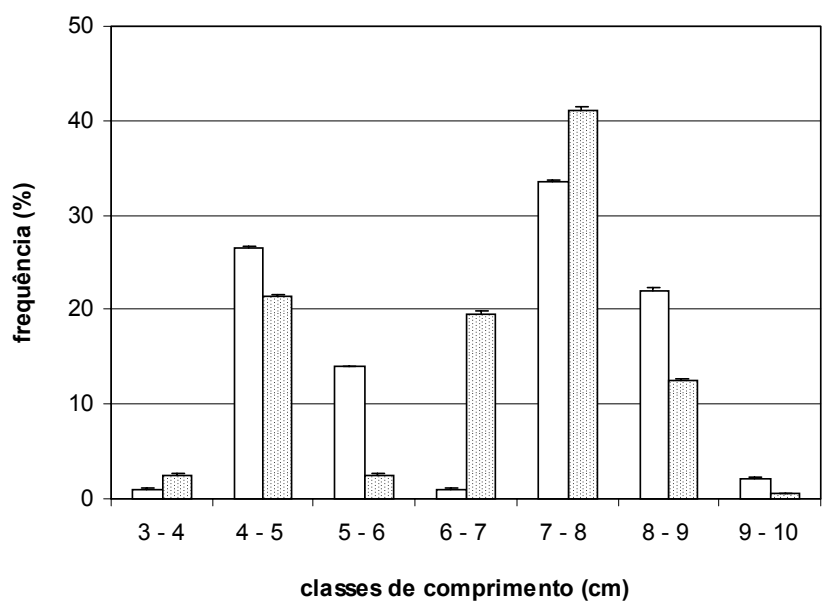

Figura 2. Distribuição trimestral das freqüências de fêmeas e machos por classe de comprimento total e o desvio padrão durante o período de estudo.

Os resultados obtidos mostram que os machos atingem comprimentos superiores aos das fêmeas. Esse comportamento pode ser explicado, em parte, pela economia da elaboração dos processos sexuais. Ao que parece, o desenvolvimento dos testículos está associado mais ao crescimento do peixe do que do ciclo anual. Isto pode ser explicado devido os machos crescerem mais rapidamente que as fêmeas nos primeiros anos de vida, assim se supõe que, para as fêmeas, ocorre maior desenvolvimento durante o ciclo reprodutivo, levando-se a comprimentos assintóticos menores (AgostinHo et al.1986).

A equação obtida para a relação peso total $\left(\mathrm{W}_{\mathrm{t}}\right)$ e comprimento total $\left(\mathrm{L}_{\mathrm{t}}\right)$, a partir de todos os dados coletados e representado através dos seus valores médios, foram analisados conjuntamente para machos e fêmeas, representada pela equação: $\mathrm{W}_{\mathrm{t}}=0,0291 \mathrm{~L}_{\mathrm{t}}^{2,59}$ (Fig. 3).

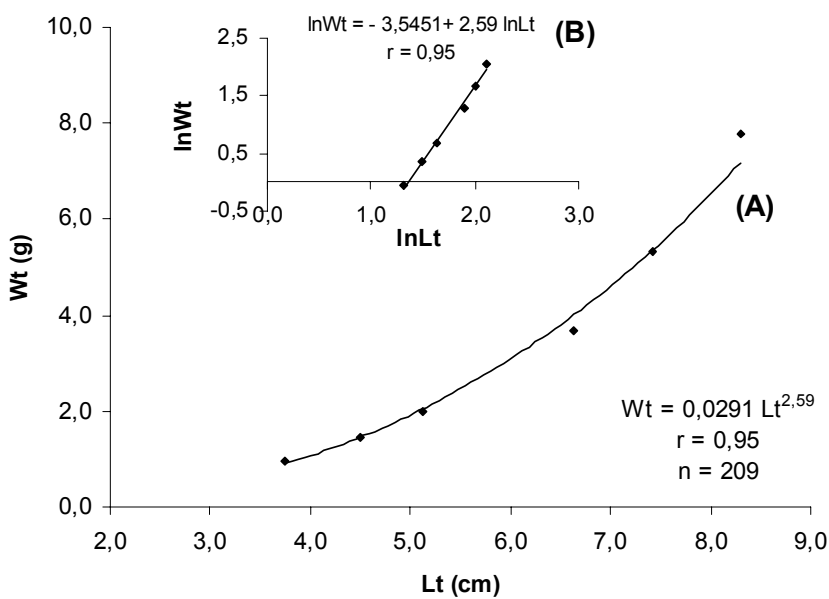

Figura 3. Curva ajustada aos pontos empíricos do peso total e comprimento total dos sexos agrupados, considerando o período total de estudo.

ORSI et al. (2002) informam que, com o coeficiente angular é possível determinar o tipo de crescimento da espécie. Se $\theta$ for igual a 3, então o crescimento é isométrico; se for maior que 3, é alométrico positivo; e se for menor que 3, é alométrico negativo. Esclarecem ainda os referidos autores que, se o crescimento é isométrico, o incremento em peso acompanha o crescimento em comprimento, mas se é alométrico negativo, há um incremento em peso menor do que em comprimento; e, se é alométrico positivo, há um incremento em peso maior do que em comprimento.

Baseado nestas informações e diante do valor do coeficiente angular $(\theta=2,59)$, conforme a relação peso total/comprimento total (Fig. 3), obteve-se para $A$. fasciatus um crescimento do tipo alométrico negativo. Resultados semelhantes foram descritos por BARBIERI et al. $(1982,1996)$ estudando a mesma espécie na Represa do Lobo (Ecossistema Broa, São Paulo).

$\mathrm{O}$ parâmetro $\theta$, da mesma forma que as variáveis peso $\mathrm{x}$ comprimento podem também variar para peixes de uma mesma espécie, mas dentro de alguns limites, de acordo com a localidade, comprimento médio da população, idade. Portanto (Verani 1980, in Gurgel \& Mendonça 2001) informa que estas variáveis podem estar condicionadas a diferenças do ambiente e genéticos da espécie.

Revista Brasileira de Zoologia 21 (1): 131-135, março 2004 
O estudo populacional de peixes analisados através dos índices biométricos, por ser uma ferramenta importante quando se pretende estabelecer programas de conservação e normatização da pesca tem recebido atenção especial dos especialistas. Estes parâmetros, segundo recomendação de Gulland (1977), devem ter um significado biológico e serem facilmente incorporados aos modelos matemáticos.

Os valores médios trimestrais do fator de condição, índice gônadossomático associados aos índices pluviométricos são apresentados na figura 4 . Observa-se que, o IGS tende a aumentar a partir do trimestre nov-dez-jan, alcançando seus maiores valores no trimestre seguinte, para as fêmeas e os machos, coincidindo com a elevação das médias dos valores de precipitação pluviométrica, enquanto que, o fator de condição tende a apresentar os valores médios inferiores neste período.

A determinação da época de reprodução e o período em que os exemplares se encontram em melhores condições alimentares têm sido investigados através da análise dos parâmetros da biologia pesqueira (GURGEL et. al. 2000). O fator de condição pode ser definido como o estado de bem estar do peixe, ou seja, como o animal aproveita os recursos disponíveis existentes numa determinada época do ano. É freqüentemente utilizado como um indicador do período de desova, uma vez que neste período a intensidade alimentar pode cessar e o fator de condição mostrar valores inferiores (BARBIERI et al. 1996)

Evidencia-se na variação trimestral do IGS (Fig. 4) que fêmeas e machos de $A$. fasciatus apresenta um período de reprodução longo, revelando maior atividade reprodutiva no trimestre fevereiro-março-abril de 1997, coincidindo com o aumento da precipitação pluviométrica enquanto que, o fator de condição neste mesmo período encontra-se em declínio. A queda do fator de condição pode estar relacionada ao uso das reservas do corpo para o processo de desenvolvimento gonadal (Gurgel et al. 1991). GuRgel et. al (1997) destacam ainda que o fator de condição pode sofrer alterações em função dos fatores intrínsecos (reservas orgânicas, desenvolvimento gonadal e tamanho dos exemplares) e extrínsecos (disponibilidade alimentar, temperatura, fotoperíodo, entre outros).

Os resultados estão de acordo com os obtidos por BARBIERI \& Barbieri (1988) e Barbieri et al. (1996), para esta mesma espécie em outra região (Represa do Lobo), apresentando desova total com maior atividade reprodutiva nos bimestres outubronovembro e dezembro-janeiro.

\section{AGRADECIMENTOS}

Ao CNPq, pela concessão do auxílio à pesquisa (Processo $\left.n^{\circ} 465195 / 00-8\right)$. Ao Professor Dr. Wagner Franco Molina do Departamento de Biologia e Genética, UFRN e à Dra. Elizabeth Romagosa do Instituto de Pesca de São Paulo, pelas contribuições ao texto. Ao acadêmico Felippe Dias Lucas, do Curso de Ciências Biológicas, UFRN, pelo valioso auxílio na confecção de figuras.
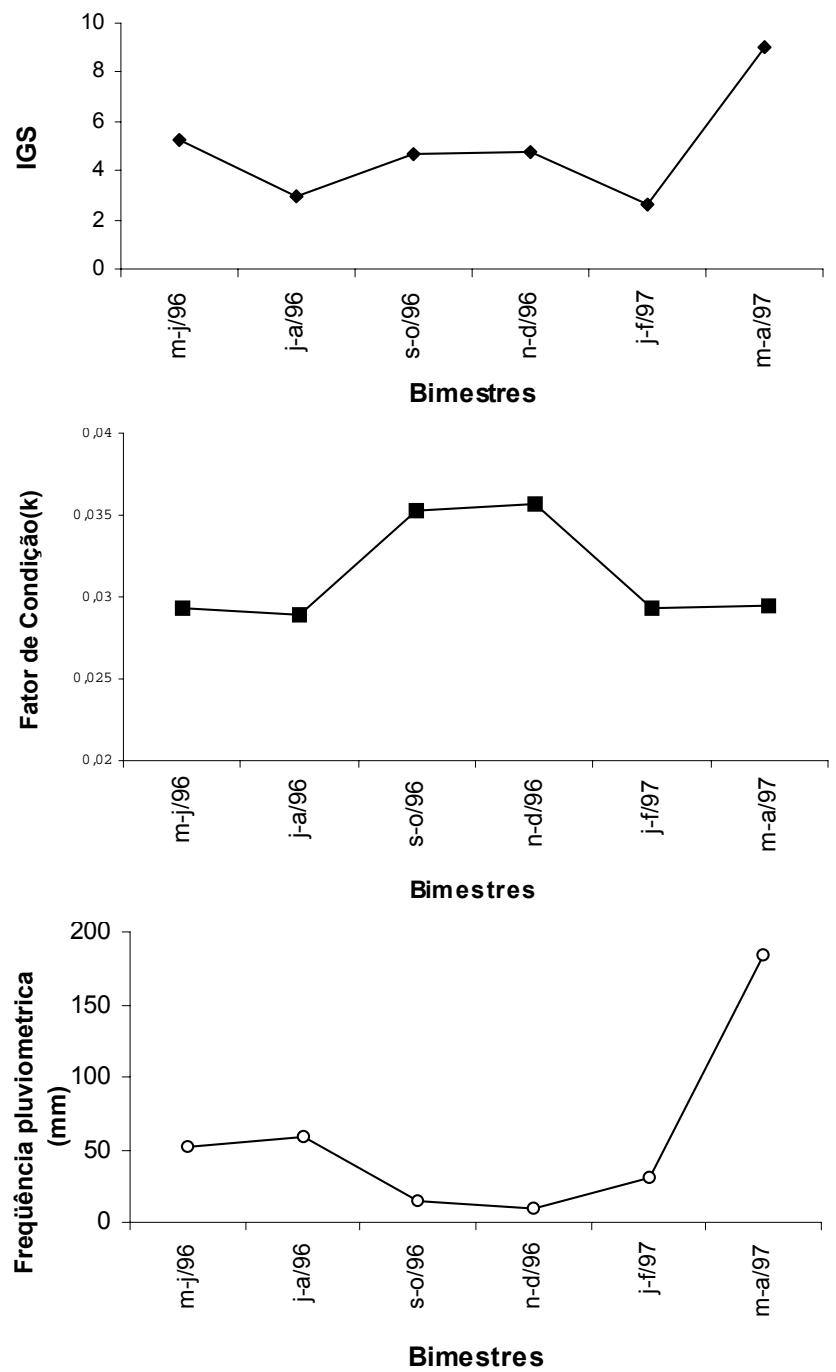

Figura 4. Valores médios trimestrais, do índice gônadossomático (IGS), do fator de condição de fêmeas e machos e freqüência pluviométrica durante o período de estudo.

\section{REFERÊNCIAS BIBLIOGRÁFICAS}

Agostinho, A.A.; G. Barbieri; J.R. Verani; C.S. Agostinho. 1986. Ciclo reprodutivo e primeira maturação de Rhinelepis aspera (Agassiz 1829), (Teleostei, Loricariidae) no rio Paranapanema. Revista Unimar, Maringá, 8 (1): 17-27.

Barbieri, G.; E.P. SAntos, 1980. Dinâmica de nutrição de Geophagus brasiliensis (Quoy e Gaimard, 1824), na represa do Lobo, Estado de São Paulo, Brasil. Ciência e Cultura, São Paulo, 32 (1): 87-89.

BARBIERI, G. \& M.C. BARBIERI. 1988. Curva de maturação, tamanho de primeira maturação gonadal e fecundidade de Astyanax bimaculatus Linnaeus, 1758 e Astyanax fasciatus Cuvier, 1819, 
da Represa do Lobo, Estado de São Paulo (Osteichthyes, Characidae). Revista Ceres, Viçosa, 35 (197): 64-77.

BARbieri, G.; M.V. SANTOS \& J.M. SANTOS. 1982. Época de reprodução e relação peso/comprimento de duas espécies de Astyanax (Pisces, Characidae). Pesquisa Agropecuária Brasileira, São Paulo, 17 (7): 1057-1065.

Barbieri, G.; S. Hartz \& J.R. Verani. 1996. O fator de condição e índice hepatossomático como indicadores do período de desova de Astyanax fasciatus Cuvier, 1819, da Represa do Lobo, São Paulo (Osteichthyes, Characidae). Iheringia, Ser. Zoolo. Porto Alegre, (81): 97-100.

BRITSKI, H.A. 1964. Sobre uma nova espécie de Astyanax do Rio Mogi-Guassu (Pisces, Characidae). Papéis avulsos Zool., São Paulo, 16: 213-215.

Câmara, J.J.C.; A.M. Rodrigues; E.C. Campos; R.A. Santos \& J. Mandelli. 1991. Pesca seletiva do tambiú, Astyanax bimaculatus Linnaeus, 1758 (Characiformes, Characidade), com a utilização de redes de emalhar, na represa de Ibitinga, rio Tietê, Estado de São Paulo, Brasil. Boletim do Instituto de Pesca, São Paulo, 18: 51-60.

Dieb, J.; H.C.B. Gurgel \& G. Barbieri. 1991. Aspectos do comportamento biológico do "Jacundá", Crenicichla lepidota (Heckel, 1840) (Perciformes, Cichlidae) da Lagoa do Bonfin. Nízia Floresta/RN. Anais do Seminário Regional de Ecologia, São Carlos, 6: 253-270.

GÉRY, J. 1977. Characoids of the world. Neptune City, T.F.H. Publications Inc., 672p.

Godoy, M.P. 1959. Age, growth, sexual maturity, behavior, migration, tagging and transplantation of curimbatá (Prochilodus scrofa Steindachner, 1881) of Mogi Guassú river, São Paulo State, Brasil. Anais da Academia Brasileira de Ciências, São Paulo, 31: 447-477.

GulLAND, J.A. 1977. Fish populations dynamics. London, John Willey \& Sons, 372p.

Gurgel, H.C.B. 1995. Estrutura da população de Metynnis cf. roosevelti Eigenmann, 1915 (Characidae, Myleinae), da Lagoa Redonda, Nísia Floresta, Rio Grande do Norte, Brasil. Boletim do Instituto de Pesca, São Paulo, 22 (1): 61-71.

Gurgel, H.C.B. \& V.A. Mendonça. 2001. Estrutura populacional de Astianax bimaculatus vittatus (Castelnau, 1855) (Characidae, Tetragonopterinae) do Rio Ceará Mirim, Poço Branco, RN. Revista Ceres, Viçosa, 48 (276): 159-168.

Gurgel, H.C.B; G. Barbieri; J.A. Pereira \& J.R. Verani. 1991. Estrutura populacional e variação do fator de condição do bagre amarelo, Arius luniscutis Cuvier e Valenciennes, 1840
(Siluriformes, Ariidae), do estuário do rio Potengí (Natal/ RN). Anais do VI Seminário Regional de Ecologia, São Carlos, 6: 237-252.

Gurgel, H.C.B; G. Barbieri \& J.R. Verani. 1997. Análise do fator de condição de Metynnis cf. roosevelti Eigenmann, 1915 (Characidae, Myleinae) da lagoa Redonda, Município de Nísia Floresta, Rio Grande do Norte, Brasil. Anais do VIII Seminário Regional de Ecologia, São Carlos, 8: 357-376.

Gurgel, H.C.B.; C.Q. Albuquerque; D.S.L. Souza \& G. Barbieri. 2000. Aspectos da biologia pesqueira em fêmeas de Cathrops spixii Spix \& Agassiz, 1829 do estuário do rio Potengi, Natal/RN, com ênfase nos índices biométricos. Acta Scientiarum, Maringá, 22 (2): 503-505.

Nikolski, G.V. 1969. Theory of fish population dynamics. Edinburgh, Oliver and Boyd, 352p.

Nomura, H. 1975. Alimentação de três espécies de peixes do gênero Astyanax Baird \& Girrard, 1854 (Osteichthyes, Characidae) do Rio Mogi Guassu, SP. Revista Brasileira de Biologia Rio de Janeiro, 25 (4): 595-614.

Orsi, M.L.; O.A. Shibatta \& A.T. SiLva-Souza. 2002. Caracterização biológica de populações de peixes do rio Tibagi, localidade de Sertanópolis, p. 425-432. In: M.E. MedRi (Ed). A Bacia do Rio Tibagi. Londrina, Universidade Estadual de Londrina, 595p.

Raposo, R.M.G. \& H.C.B. Gurgel. 2001. Estrutura da população de Serrasalmus spilopleura Günther, 1864 (Pisces, Serrasalmidae) da lagoa de Extremoz, Estado do Rio Grande do Norte, Brasil. Acta Scientiarum, Maringá, 23 (2): 409-414.

Rebouças, R. 1964. Notas preliminares sobre a biometria de Astyanax fasciatus (Pisces, Characidae). Papéis Avulsos de Zoologia, São Paulo, 16: 27-44.

SANTOS, E.P. 1978. Dinâmica de populações aplicada à pesca e piscicultura. São Paulo: Hucitec, 129p.

Santos, M.T.D.; M.T.D. Giamas; E.C. Campos; J.J.C. CÂmara \& H. Vermulum Jr. 1995. Dinâmica da nutrição do tambiú Astyanax bimaculatus Linnaeus, 1758 (Pisces, Characiformes, Characidae), na represa de Ibitinga, Estado de São Paulo, Brasil. Boletim do Instituto de Pesca, São Paulo, 22 (1): 115-124.

VAnzolini, P.E.; R. RebouçAs \& H.A. BRitSKi. 1964. Caracteres morfológicos de reconhecimento específico em três espécies simpátricas de lambaris do gênero Astyanax (Pisces, Characidae). Papéis Avulsos de Zoologia, SãoPaulo, 16: 267-299.

VAzzoler, A.E.A.M. 1996. Biologia da Reprodução de Peixes Teleósteos: Teoria e Prática. Maringá, Editora Universidade Estadual de Maringá, 169p.

Recebido em 10.Il.2003; aceito em 27.II.2004. 